\title{
Virtualized Resource Allocation Algorithm in Mobile Internet
}

\author{
Feng-Zhao ${ }^{1}$, Jing-Chen ${ }^{2}$ \\ ${ }^{1,2}$ Shandong Labor Vocational and Technical College Jinan, China \\ zhaofeng@sdlvtc.cn ${ }^{1}$,chenjing@sdlvtc.cn ${ }^{2}$
}

\begin{abstract}
Mobile Internet has its own terminal access at any time, limited bandwidth and other characteristics, the effective allocation of resources and management is the primary means to improve the cloud computing performance. However, due to the diversification of its businesses, small service scheduling granularity and flexibility, for this feature, we propose resource allocation prediction method by SVM and wavelet decomposition, effectively improve the performance of the system.
\end{abstract}

Keywords: mobile cloud computing, virtualized resources, support vector machines, wavelet decomposition, cloud computing

\section{Introduction}

With the advancement of mobile access technology and mobile terminals, mobile Internet ${ }^{[1]}$ business has been developed rapidly. In the next few years, with the rapid development of $4 \mathrm{G}$ network large-scale commercial, PDA, smart phones, home digital center, tablet PCs and other mobile terminals, mobile Internet will continue to maintain a rapid development, leading the IT industry and the Internet forward progress. However, the explosive growth of mobile Internet services, the demanding of users experience have become increased, massive mobile applications flooding the market, user demand for services is also faster, more stable and more practical. In addition, mobile terminals security and mobile access network security issues have become increasingly prominent.

In recent years, cloud computing ${ }^{[2]}$ has become a hot topic of the Internet industry. The integration of high performance computing, grid computing, cluster computing and distributed computing technology, the cloud computing network can use the existing resources, to provide reliable and efficient network services. According to Gartner released the 2012 top ten strategic technology trends report, cloud computing has become the most cutting-edge technology, it has changed the world many organizations and businesses mode of operation.

As the combined product of cloud computing and mobile Internet, mobile cloud computing has become a new hotspot. Cloud computing dynamic management of virtual resources $^{[3]}$, to focus on virtualization resources, on-demand features, mobile Internet business can achieve stable and efficient services. Mobile cloud computing can solve the problem that computing and storage capacity of mobile Internet applications. In addition, mobile cloud computing can eliminate services geographic restrictions, realize the features of cloud data sharing and efficient processing real-time business.

Virtualized resources management is an important aspect of cloud computing research, which has become a great concern. It utilizes resource virtualization and virtual machine technology, the cloud computing platform as a support, research how to optimize virtualized resource management to improve resource utilization of cloud computing, expand mobile Internet services and reduce energy consumption. Compared with the traditional cloud computing, due to many limitations exist in terms of bandwidth, the terminal performance and application characteristics in mobile cloud computing, virtualized resource management process becomes very complicated, this should consider 
multiple levels resource entities and then complete the more fine-grained management operations. This includes the service SLA metrics, dynamic service request ${ }^{[4]}$, load balancing, virtual machine performance guarantees, power control, resource utilization and dynamic forecasting resource requests and other requirements. So virtualization resource management technology for mobile cloud computing is very significant for a study.

\section{Related Works}

Currently, more mature cloud platform mainly Google, Amazon and Baidu as the representative. Google as a cloud computing pioneer platform, with its low-cost, efficient cloud computing platform ahead of other companies, it is the another breakthrough invention after page ordering. Google will assemble a lot of ordinary PC into the cloud, build efficient, reliable computing services, Google search engine is one of application examples. Google currently uses its own server efficiency of $70 \%$ to $80 \%$, but the traditional carriers are only $15 \%$ to $30 \%$. After the application of cloud computing, Google company will reduce its operational costs to one-thousandth of the original, reducing storage costs to $1 / 60$, the overall operating costs has become half of the original. Google has introduced high-performance computing, big data, parallel and distributed computing resource management, distributed file system-related technologies, which play a role as a global reference of cloud calculation, as the current most widely used cloud computing platform. Recently, Google has launched a Linux-based browser system Chrome OS, to provide users with a wide range of services through a cloud platform to create a "network as the core " new man-machine experience model ${ }^{[5]}$.

After the original platform upgrade and optimization, Amazon launched elastic cloud computing platform, which utilizes corporate infrastructure resources to provide users with remote cloud services. By virtualized network security protocol, Amazon can provide users with efficient, easy to use, flexible billing resource rental service, we established a AWS cloud platform model as a EC2 core.

As cloud computing technology becomes more mature, a lot of IT companies are beginning use cloud computing applications. In addition to Google and Amazon, such as Internet service providers, and emerged with Verizon and AT \& T as the representative of telecom operators, EMC and Cisco as the representative of the equipment suppliers, HP and IBM as the representative of the integrated systems suppliers, SaleForce and Microsoft represented software vendors.

Currently, the mobile cloud computing is still in development and investigation. But also it has a successful business case worth considering. In 2008, Apple introduced MobileMe cloud computing and cloud storage solutions, instead of the original Mac service. Mac service only service existing Mac Book service, however, MobileMe service has been greatly expanded, it can take a service to the iphone, Ipad and ipod touch and other devices. In addition, Apple has pioneered the application store Apple Store model for users and developers with cloud services-based application platform, now has more than hundreds of thousands of applications, and downloads more than 4 billion times.

As represented by Google's Android, the company gradually developed in the Tablet PC and smart phone market mobile the terminal, mobile cloud computing cloud-based Google application platform also increasingly popular, which include:

1) mobile positioning and mobile maps. Based on Google $\mathrm{Maps}^{[6]}$, users can get real-time location services, to provide users with directional line inquiry service, but also to tell the user's car, public transportation, restaurants and other surrounding service information.

2) mobile search. Integrating the traditional Internet search business, optimizing search results feedback, which allows mobile phones and other mobile devices easier to navigate. 
3) Google Street View of android version. Google unique service, allowing users to use the phone to record the surrounding scenery, easy to communicate with friends.

4) language search. Google's cloud-based platform and massive data centers and efficient processing power, can achieve more intelligent multi-language accent, voice recognition, provide a very accurate search service.

In 1959, Christopher Strachey in their academic report for the first time put forward the concept of virtualization, virtualization technology with the development of computers has been steadily progressing. Early in the 1960s, virtualization technology has been applied to IBM mainframe systems, to the 1970s, System of 370 series increasingly popular. Virtual machine monitor, can achieve physical hardware virtualization and abstraction. Now, with cluster computing, multi-core technology, widely used cloud computing and distributed computing, virtualization technology show a great advantage. It not only improved system resource utilization, safety performance, but also reduces the energy consumption and operating costs of IT companies.

In computer science technology, virtualization not only refers to the virtual machine, it mainly refers to the abstraction of computer resources, used and management centralizely. In general, the computer system includes an application API interface to the operating system, the underlying physical hardware, applications and the operating system. Virtualization technology build a virtual intermediate layer to communicate the upper and lower levels, improve the flexibility of a computer system, reducing the coupling of the lower layer. For example, the physical memory abstraction, so that the virtual memory technology emerges, so that the application considers itself a continuous address space available to solve the problem of insufficient physical memory; Through storage resources abstraction, virtual memory technology emerges, by application we achieve a multi-tasking multi-user shared file operations. Virtualization technology is mainly divided into resource virtualization, application virtualization, and platforms virtualization.

Virtualization resource management ${ }^{[7]}$ is not based on resources geographical location to achieve. That is, it is a logical view of storage, data, computing power, network resources, not physical view. For the user, through virtualization, resource management, can simplify the process of resource use and does not need to focus on the distribution and implementation of physical resources. For service providers, the use of resource virtualization management, resource management can be unified, simplified service process, efficient completion of user requests, improve service efficiency.

For virtualization, consumers can be an application, an end user, or a secondary service access. The resource is a way to provide the functionality of the service implementation, it can be normalized output and input. Where resources can be a wide variety of hardware, such as CPU, disk, server, memory, and other special equipment or networks; it can be a variety of software, such as web services and mail services.

Users use virtualized resource management model interface to access resources. The use of standardized interfaces, can reduce the coupling between users and IT infrastructure, so that when the resource changes, users will also be affected to a minimum. In addition, when the underlying resources change, run the software in a virtualized environment can take advantage of the patch, so do not need to be upgraded. By coupling relationship, administrators can minimize the impact on users under the premise, effectively managing the underlying infrastructure.

In cloud computing, the underlying infrastructure through virtualization can be abstracted into network resources, computing resources, storage resources. While the infrastructure and the different service providers are different, but virtualized resources are often already included under the functions: 1)Resource Monitor 2) Resource Abstraction 3) Data Management 4) Load Management 5) Dynamic Deployment of Resources . 


\section{Proposed Scheme}

In a virtualized resource allocation problem, the application of forecast technology can improve allocation accuracy and allocation allocative efficiency. Based on previous research and analysis on system resources, establish specific forecast model can be achieved effectively predict within the next point time resource usage.

\subsection{Times-Series Studies}

Time series refers to a chronological arranged the random sequence. Analyzed by the use of resources for cloud services, then find some time series, used to describe the characteristics and features of service resources to manage and forecast resources.

According stationary of time series ${ }^{[8]}$, time series models can be divided into non-stationary time series and stationary time series. Stationary time series could be the simplified by the prediction model, but this assumption is often only apply in the case of short-term small-time scale; however, due to the time scale is large, in fact, resource forecasting models tend to show non-stationary time series.

\section{1) The stationary time series}

Time series can often be used by $W_{t}$ to indicate that it is a collection of the probability space a set of random variables, it can be expressed as $\left\{W_{t}, t=0, t= \pm 1, t= \pm 2, \ldots\right\}$. Now, the majority of researchers, mainly research in the economic field and the non-stationary and stationary time series analysis. Smooth characteristics mainly refers to the time series of random variables, for statistical regularity of its past evolution will not change for some time in the future, namely its mathematical variance and mathematical expectation does not change. Independent of its related functions and time node, and just relate to the length of the time interval.

Establishment of stationary time series model includes model parameter estimation, model order, pattern recognition and other steps.

Identify the model there are three main ways:

a) the use of long autoregressive method, the choice of AR (P) were approaching;

b) based on the sample from the trailing end of the correlation function and to judge;

c) the use of auto-regressive moving average model;

d) There are model order four ways:

e) F test given by degrees;

f) the residual variogram order determination method;

g) the best criterion function given by degrees;

h) the relevant means.

i) Model parameter estimation There are two main methods:

j) long autoregressive method;

k) nonlinear minimum two estimates multiplication algorithm.

2) Non-stationary time series

Compared with the time series, the presence of non-stationary time series trend and periodicity, data between relevant. In addition, nodes at different times, the sequence variance and expectations are different. Because cloud application resource usage trends and there is often no regularity, therefore, the use of non-stationary time series model is more appropriate than the stationary time series model.

Although the non-stationary time series represents the trend can be a good use of resources and cyclical, but this will increase the complexity of the analysis and research. Therefore, the non-stationary time series into a stationary time series, such an analysis will be easier, so you can take time series into smoothing.

The first step of the process flow smoothly is to determine whether the sequence of non-stationary time series. Verify that the non-stationary time series, first check whether it 
is necessary to strictly distribution functions at different times of the same. If you relax the test conditions, it will have two main testing methods:

a) Characteristic root tests

For the time series modeling, calculating model parameters characteristic roots of the characteristic equation, characteristic root and then use the equation to determine whether the non-stationary. If the absolute value of the characteristic roots of less than 1 , it can be judged that the time series is non-stationary.

If judged as the non-stationary time series, then you can use the smoothing method, which was transformed into stationary time series. So the existence of smoothing methods are mainly two:

Difference Method. Non-stationary time series $W_{t}$ can be a differential operation, remember to do $\nabla W_{t}$, then there

$\nabla W_{t}=W_{t}-W_{t-1}$

Under normal circumstances, the differential operation can eliminate the linear trend time series. If you do not feature a smooth non-stationary time series belong to the linear trend, then after a difference the sequence is stationary.

Seasonal difference. Different with differential method, the role of seasonal differencing is mainly used to eliminate the cyclical trend. Cyclical trends in the main is in accordance with a certain period of time $T$ for sequence-cyclical, namely that the time interval $T$, at different times, there is some linkages between data. By doing differential operation across the cycle, it may be eliminated between data correlation.

It can be expressed $\nabla$ as a differential season, so there $\nabla_{T}=W_{t}-W_{t-T}$, which is at periodic intervals $T$.

b) partial autocorrelation function and autocorrelation function test methods

The time series of zero-mean process, according to the partial autocorrelation function and self-tailing related functions and truncation of the judge. If the partial autocorrelation function and autocorrelation function, neither censored nor tailing, it may determine whether the non-stationary time series properties.

\subsection{Resources Allocate Prediction Algorithm by Wavelet and Support Vector Machines ${ }^{[9]}$}

1) the definition of wavelet

Wavelet can be defined as: Suppose $\Phi(t)$ is a square integrable functions, viz $\Phi(t) \in L^{2}(R)$. If the Fourier transform satisfies the following conditions

$\int_{R} \frac{|\Phi(w)|^{2}}{w} d w<\infty$

It may be referred to mother wavelet $\Phi(t) . \Phi(t)$ can be scaled and translated to give:

$\Phi_{a, b}(t)=\frac{1}{\sqrt{a}} \Phi\left(\frac{t-b}{a}\right)$

It may be referred $\Phi_{a, b}(t)$ to the wavelet function, $a>0, a, b \in R, a$ is called the stretch factor, $b$ known as the translation factor.

2) DWT Discrete Wavelet Transform

The discrete-time wavelet transform is often used for frequency analysis and numerical analysis. Discrete-time wavelet transform refers to the input and output signals are discrete. It can be defined as:

$D W T(m, n)=a \int_{0}^{\frac{\pi}{2}} f(t) \Phi\left(a_{0}^{m} t-n b_{0}\right) d t$ 
Among them, $a=a_{0}^{-m}, b=n b_{0} a_{0}^{-m}, m$ and $n \in z$, and $a_{0}$ are $b_{0}$ constants.

3) Wavelet decomposition process

Suppose resource usage input sequence is $W(t), g(k)$ is wavelet transform high-pass filter, $f(k)$ is a low pass filter wavelet transform, wavelet decomposition of series is $K$, the wavelet decomposition virtual machine resource usage can be expressed as:

a) Initialization.

Let $c_{0}=W(t), f_{1}(k)=f(k), g_{1}(k)=g(k), i=1$ 。

b) Wavelet decomposition.

$$
\begin{aligned}
& c_{i+1}[k]=c_{i}[k] * f_{i+1}[-k] \\
& l_{i+1}[k]=l_{i}[k] * g_{i+1}[-k]
\end{aligned}
$$

Which $*$ represents the convolution operation.

c) The filter of the insertion zero process.

$$
\begin{aligned}
& f_{i+1}[k]=\left\{\begin{array}{c}
0, i \text { is an odd number } \\
h_{i}\left[\frac{k}{2}\right], i \text { is } \text { an even number }
\end{array}\right. \\
& g_{i+1}[k]=\left\{\begin{array}{c}
0, i \text { is an odd number } \\
g_{i}\left[\frac{k}{2}\right], i \text { is an even number }
\end{array}\right.
\end{aligned}
$$

d) Carry out $i=i+1$, if $i \geq K$, then the operation is terminated, otherwise return step b) proceed to wavelet decomposition.

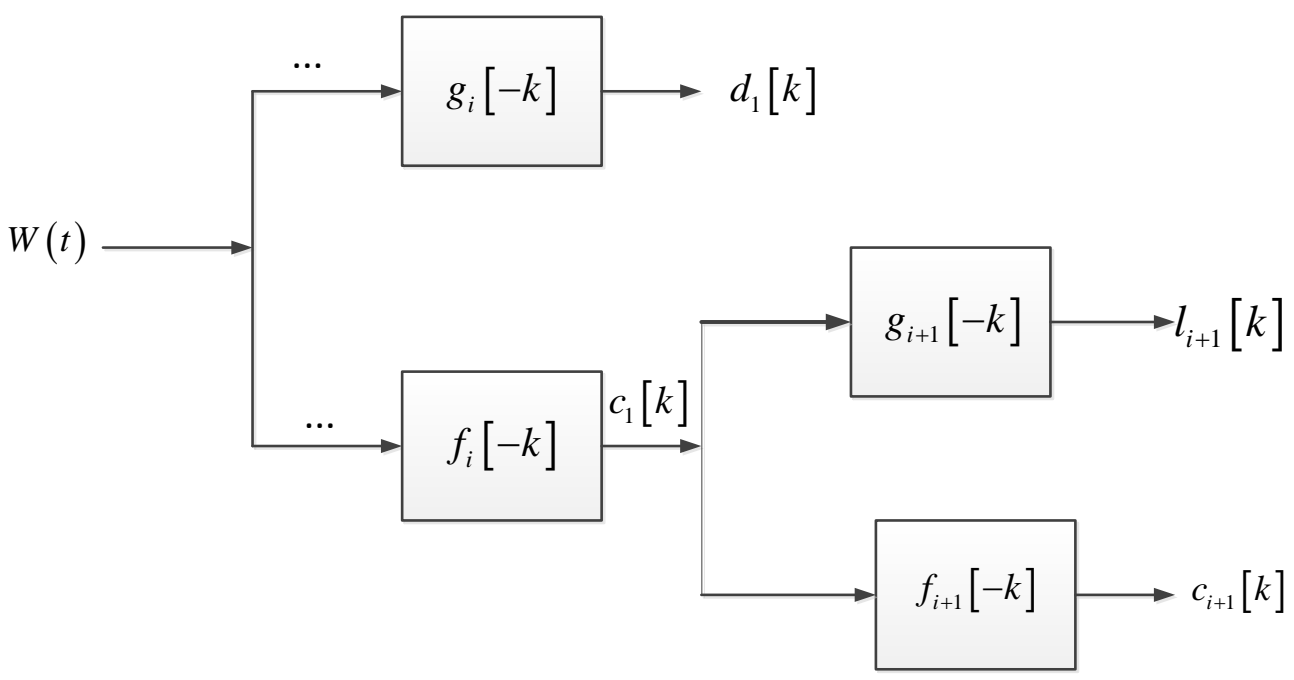

Figure 1. Wavelet Decomposition

According to the wavelet decomposition of Figure 1 may know, in the virtual host resource usage wavelet transform, the component of each scale can be broken down, which also provides the basis for follow-up support vector machine.

4) Support vector machine

Support vector machine is a supervised learning method, which has been applied to a large number of the statistical classification and regression analyzed. Support vector machine is a generalized linear classifier, which belongs to a special case Celtics Love standardized methods. Linear classifier can be minimized in the case of error experience, but also to maximize the geometric fringe. Therefore, support vector machine is also known maximize Fringe classifier. Traditional machine learning algorithms and compared 
with non-linear support vector machine learning ability, and good generalization performance, more suitable for nonlinear, time-varying resource usage prediction.

For the support vector machine, kernel functions commonly used are:

a) polynomial kernel $K(x, y)=[(x \bullet y)+1] d$;

b) linear kernel $K(x, y)=x \bullet y$;

c) Layer neural network kernel $K(x, y)=\tanh (a(x \bullet y)+b)$;

d) radial basis function $K(x, y)=\exp \left(|x-y|^{2} / d^{2}\right)$.

Assuming component $W_{t}$ for access to resources, then the resource forecasting model based on support vector machine can be expressed as:

$w_{t}=S V M\left(w_{t-1}, w_{t-2}, \ldots, w_{t-s}\right)$

Which $s$ represents the embedded dimensions, SVM support vector machine model.

5) Prediction algorithms

The predict algorithm are summarized as follows:

a) The historical data of the virtual machine resource usage collection, you will be able to obtain a discrete time series $W(t)$.

b) The time series wavelet decomposition. Wavelet decomposition scale settings, will break down the original resource usage data into components of the respective scales.

c) the normalization process each scale weight. Because of changes in the use of components of each scale is relatively large, in order to speed up the SVM prediction accuracy and machine learning pace, then we must first normalize each component, we can be expressed as:

$w_{i}^{\prime}=\frac{w_{i}-w_{\min }}{w_{\max }-w_{\min }}$

$w_{i}=w_{i}^{\prime} \times\left(w_{\max }-w_{\min }\right)+w_{\min }$

Where, the sequence of the original resource is $w_{i}, w_{i}$ is the normalized resource use sequences, $w_{\min }$ and $w_{\max }$ are used trough value and peak value of the resource.

d) SVM model initialization. The differentiation of the original data sequence test sample set and the training set, based on the embedding dimensions to determine the output vector and the input vector of SVM.

e) The SVM model and kernel function parameter selection. SVM linear kernel function kernel function, high-frequency part of the resource usage data can be approximated smooth. Data also showed low frequency of nuclear non-stationary nonlinear kernel function using polynomial function to achieve.

f) Prediction and training SVM model. The use of optimized parameters for the model training set to learn, build predictive models, we can predict the test set.

g) the predicted results of the anti-scale components of the normalization process, can be expressed using the formula as follows:

$w_{i}=w_{i}^{\prime} \times\left(w_{\max }-w_{\min }\right)+w_{\text {min }}$

h) To normalized component of each scale wavelet reconstruction, then we can get the final prediction, the algorithm can end.

\section{The Experimental Results and Analysis}

Experiment, we established in line with the experimental simulation environment cloud center, simulate user requests in real time cloud service. Suppose a virtual machine of total memory $2048 \mathrm{M}$, using a total of 1000 time node, each node time interval is 80 s, the virtual host can be collected over a period of time of peak memory resource usage. 
Figure 2 is a waveform diagram in which the Y-axis represents the peak current node memory usage, Y-axis represents time node.

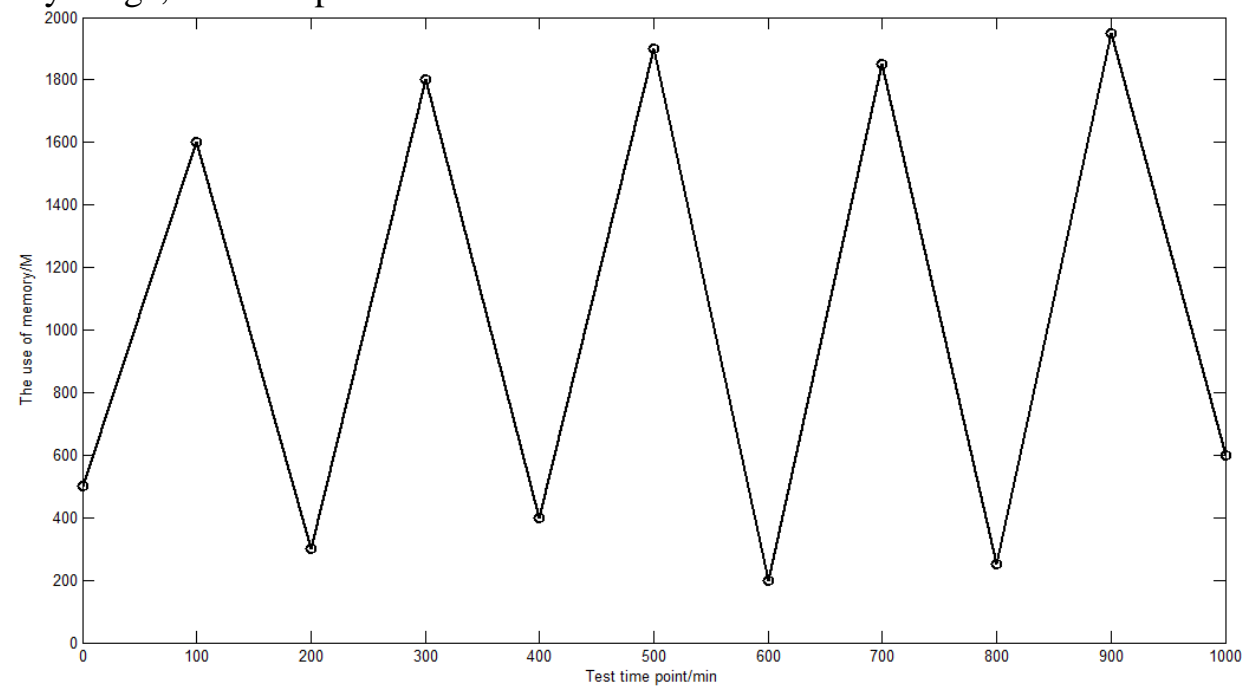

Figure 2. Original Data Sequence Diagram

It can be seen from Figure 2, the use of memory resources associated with long, nonlinearity. In this experiment, we choose as a base wavelet db2. May know, in a different dimension wavelet decomposition, the wavelet analysis of the effect and the final result will be very different prediction. So, after many experiments later, then you can select the appropriate decomposition dimension, thus improving forecast accuracy, reduce loss of information.

First, the training samples 500 data were two of $\mathrm{db} 2$ wavelet decomposition, the following results can be obtained, as shown in Figure 3.

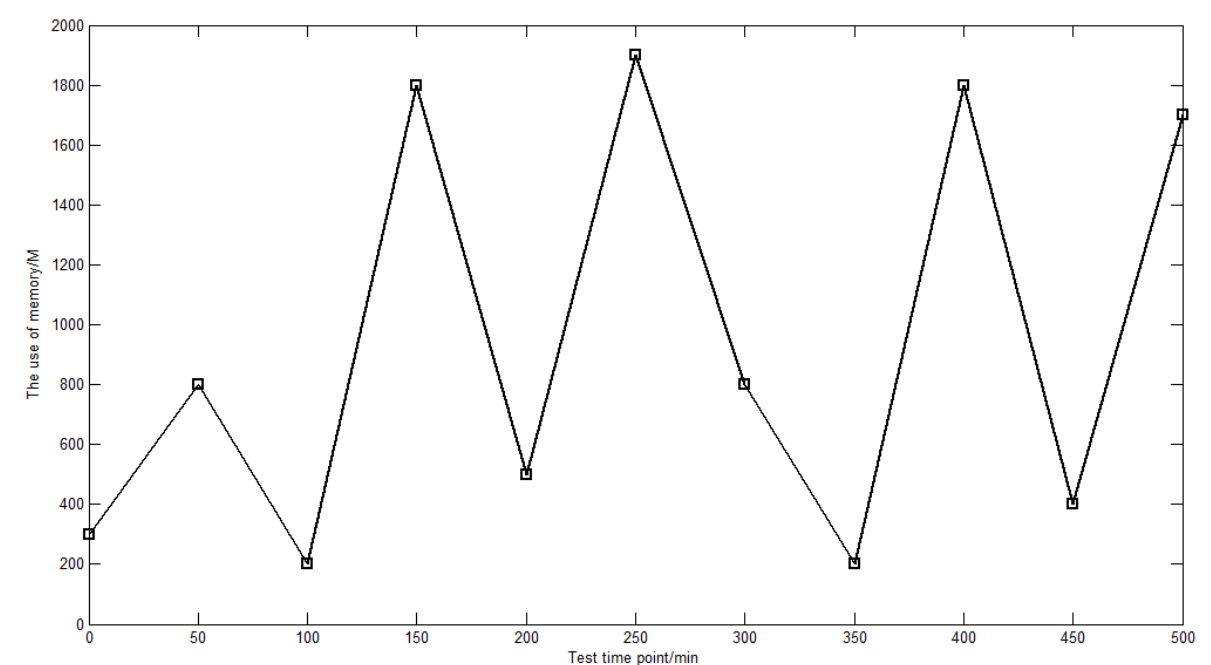

Figure 3. Two Layers Wavelet Decomposition

By decomposition, the training sample can eliminate instability, noise suppression, and establish a stationary time series model, will improve the accuracy of the final prediction. SVM modeling wavelet reconstruction, we can predict the final result obtained fitting contrast effect diagram shown in Figure 4. 


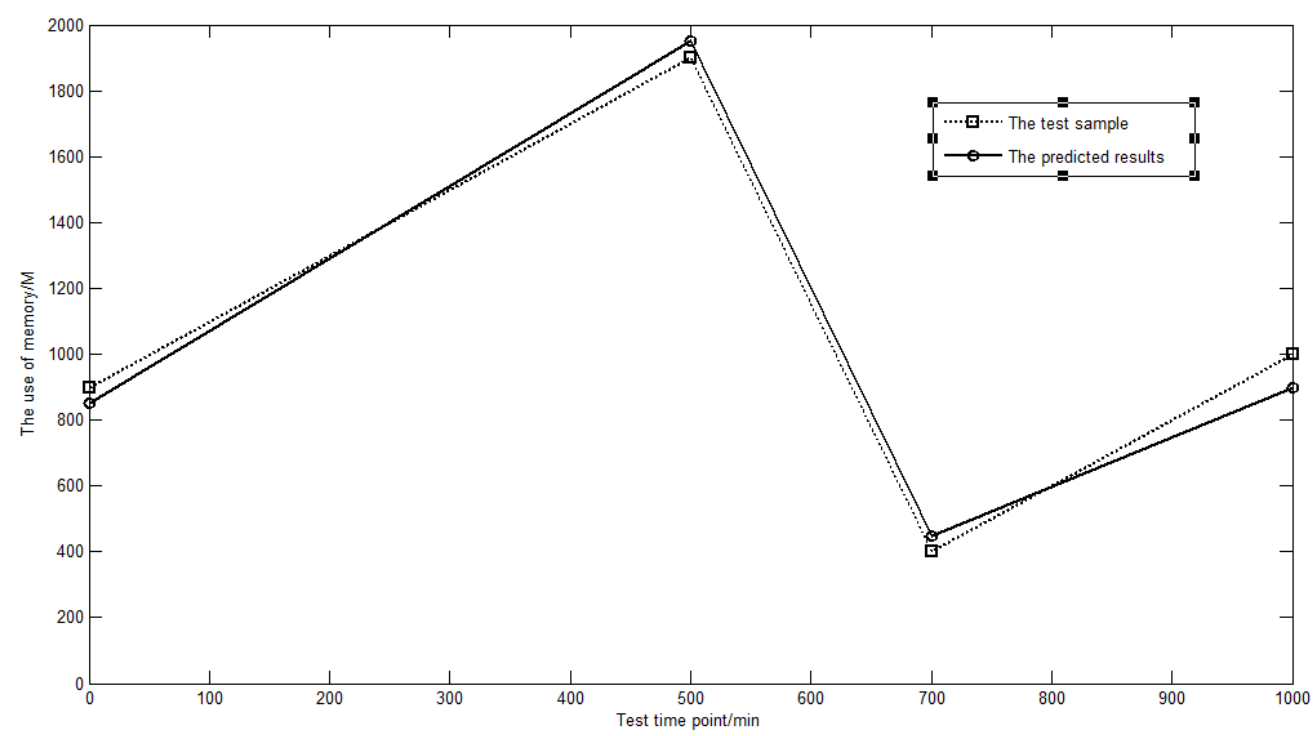

Figure 4. Test Samples and Predicted Results Comparison Chart

As seen in Figure 4, the final prediction results and collected samples is basically consistent, predictable errors within the acceptable range. SVM and wavelet combined, can more accurately describe the historical resource usage, so you can point the next time the resource prediction accuracy is more accurate.

\section{Conclusion}

This paper presents a method for allocation of resources prediction SVM and wavelet decomposition. Learning characteristics and methods of analysis of time series prediction model, through test analysis can be drawn from it can improve the performance of the mobile Internet system, fully verify the advantages of this algorithm.

\section{References}

[1] P E Pedersen, "Adoption of mobile Internet services: An exploratory study of mobile commerce early adopters [J]", Journal of organizational computing and electronic commerce, vol. 15, no. 3, (2005), pp. 203-222.

[2] M. Armbrust, A. Fox, R. Griffith, et al. "A view of cloud computing[J]", Communications of the ACM, vol. 53, no. 4, (2010), pp. 50-58.

[3] S. Adabala, V. Chadha, P. Chawla, et al, "From virtualized resources to virtual computing grids: the In-VIGO system [J]", Future Generation Computer Systems, vol. 21, no. 6, (2005), pp. 896-909.

[4] Q. Zhang, Q. Zhu, M F Zhani, et al, "Dynamic service placement in geographically distributed clouds[J].", Selected Areas in Communications, IEEE Journal on, vol. 31, no. 12, (2013), pp. 762-772.

[5] P. Falzon, R. Amalberti, N. Carbonell, "Man-Machine Voice Dialogue: The Role of Interlocutor Models[C]", //Analysis, Design and Evaluation of Man-Machine Systems 1988: Selected Papers from the Third IFAC/IFIP/IEA/IFORS Conference, Oulu, Finland, 14-16 June 1988. Elsevier, (2014): 387.

[6] M S Wilson, C T Miller, "Using Google Maps Web-application to Create Virtual Plant Maps for Use as an Online Study Tool in Plant Identification Courses[J]", HortTechnology, vol. 25, no. 2, (2015), pp. 253-256.

[7] Y. Katayama, K. Yamada, K. Shimano, et al. "Hierarchical resource management system on network virtualization platform for reduction of virtual network embedding calculation[C]", //Network Operations and Management Symposium (APNOMS), 2013 15th Asia-Pacific. IEEE, (2013), pp. 1-3.

[8] C. Chatfield, "The analysis of time series: an introduction[M]", CRC press, (2013).

[9] D. Meyer, F H T Wien, "Support vector machines [J]", The Interface to libsvm in package e1071, (2014). 


\section{Authors}
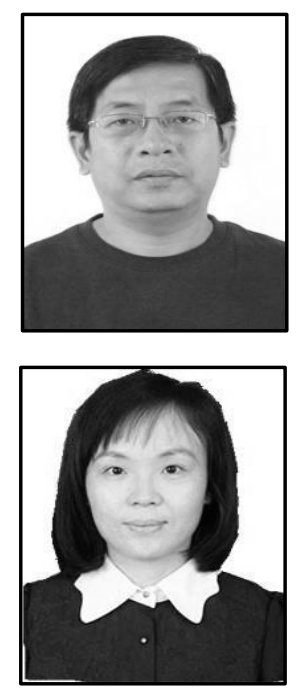

Zhao Feng. Shandong labor vocational and technical college of information engineering and art design, an associate professor, senior technicians, technical experts in shandong province.Teaching in more than 20 years, many students attended the awards, the management and provincial-level scientific research topic, Ed. A number of textbooks, published and core IE number of papers.

Chen Jing. Shandong labor vocational and technical college of information engineering and art design, an associate professor, associate professor, technicians, computer programmer. Thirteen years engaged in the computer professional teaching, guiding students to participate in national games results are excellent. Chaired provincial above topic 3 items, editor of three textbooks. 